\title{
Somatovisceral Motor Patterns in the Insula ${ }^{1,2}$
}

\author{
MARY JANE C. SHOWERS ${ }^{3}$ AND EDWARD W. LAUER \\ Central Nervous System Research Unit of the Kresge Medical Research, \\ University of Michigan Medical School, Ann Arbor, Michigan
}

This brief study was made as a corollary to previous experimental work on additional motor areas of the macaque monkey. The region of the island cortex is shown in figures $6 \mathrm{~A}$ and $\mathrm{B}$, as it relates to the opercular areas of the matching cerebrum. Although there is not general agreement as to the homology of the insula (von Bonin and Bailey, '47, Frontera, '56) of monkey with that of man, it appears that two varieties of cortex can be distinguished in both. Von Bonin and Bailey ('47) describe agranular cortex at the limen as the dominant histologic feature, although some granular cortex is found in the caudal part of the insula. Several authors (von Bonin and Bailey, '47; Kaada, Pribram and Epstein, '49 and Kaada, '51) emphasized the presence of discernible rostral motor cortex and caudal sensory cortex in the island.

Frontera's ('55) description of the macaque insula will be used as reference here. Branches of the middle cerebral artery divide the island into a dorsorostral portion and a ventrocaudal portion without there being evidence of true gyri and sulci (fig. 6A). The dorsorostral insula is covered by the frontoparietal operculum of the Walker ('40) areas 6, 4, and 1, and the ventrocaudal region by the temporal operculum of area 22 of the superior temporal gyrus (fig. 6B).

\section{REVIEW OF THE LITERATURE}

Frontera ('56) described the negative results of Grünbaum and Sherrington ('03) and Leyton and Sherrington ('17), who investigated the island of Reil in chimpanzee, orangutan and gorilla using an inductorium and bipolar electrodes. However, a number of experimental results can be tabulated that suggest the nature of an additional motor area and cortical autonomic regulator in the functional char- acteristics of the insular cortex. Ruch and Patton ('46) suggested that a taste center may be found in the precentral operculum and insula of chimpanzee. Sugar, Chusid and French ('48) found a second motor area in macaque lying on the lateral and the medial walls of the frontoparietal operculum and posterior part of the insula. Their experimental results included movements of the face, the tongue and the eyes, along with changes in respiration. Frontera ('55) obtained distinct movements of the face and the contralateral upper and lower extremities when he explored the ventrocaudal part of the insula by use of an inductorium on monkeys anesthetized with barbiturates and ether. Some of these same areas gave rise to shoulder and upper extremity movements accompanied by shivering and salivation when Frontera ('56) repeated his previous work under a somewhat different operative procedure.

Kaada, Pribram and Epstein ('49) elicited alterations in blood pressure and respiration from stimulation applied particularly to the region of the limen of the insula. Kaada ('51) extended these observations to include the anterior sylvian and ectosylvian gyri of cats and dogs. With stimulation of these gyri there were such results as closing of the eyes and pupillary dilation, piloerection, salivation, licking, swallowing and chewing movements, micturition and defecation. Wall

1 Grateful acknowledgment is given to the Parke-Davis Pharmaceutical Company for the monkeys provided for this experimentation.

2 This study was financed in part by research grants BF-7479 and B-1442 from the United States Public Health Service Division of Neurological Disease and Blindness.

3 Present address: Department of Biology, Our Lady of Cincinnati College, Cincinnati 6, Ohio.

4 Present address: Department of Anatomy, University of Michigan, Ann Arbor, Michigan. 
and Davis ('51) also found changes in respiration and blood pressure from the region of the insula close to the orbital cortex. An extensive study of the results of stimulation of the insula was carried on by Hoffman ('51) and Hoffman and Rasmussen ('53). They ascertained highly discrete changes in blood pressure, respiration and gastrointestinal tone during the stimulation of the insula. Livingstone ('55) included the insula in his review of brain mechanisms and psychosomatic function. In the work of Penfield and Faulk ('55) there is a resumé of most of the experimental work cited above and a report of the stimulation and the removal of the insula of man, as observed over a 10-year span of time. In the last observations, along with those of Poole ('54), sensorimotor responses, which included epigastric distresses, nausea and vomiting and changes in intestinal elimination, were prominent features related to disorders in the insula of man. The clinical observations were based upon cases of epilepsy, tumors, surgery and stimulation of the temporal lobe and island of Reil.

\section{CURRENT EXPERIMENTATION}

Cortical stimulation was performed with a blunt-tipped silver wire insulated with plastic tubing and a metal rectal plug served as the indifferent electrode. A Grass stimulator (model S4B) was the source of square wave pulses with a frequency of 40 per second, duration 1 msec., and intensity of 7 to $10 \mathrm{v}$. The observations, testing, experimental procedure and microscopic technique were carried out as previously recounted in Showers ('59).

In the stimulation of the three Macaca mulatta monkeys, the right and the left insular regions were explored separately, and in a composite plan as indicated in figures $1 \mathrm{~A}, \mathrm{~B}, \mathrm{C}$. The limen, as it approached the orbital cortex, was compared with the caudal tip of the insula (figs. 1A, B). The area on either side of the middle cerebral artery was explored as shown in figures $1 \mathrm{~B}, \mathrm{C}$. The region described as dorsorostral (figs. 1A, B, C) was contrasted with the ventrocaudal area (figs. $1 \mathrm{~A}, \mathrm{~B}, \mathrm{C}$ ) in their respective responses to stimula- tion. Finally, the entire insular surface was stimulated in a serial order for both the dorsorostral and the ventrocaudal regions as shown in figure 2. Differentiation of the results obtained from excitation of the insula was made from those obtained by stimulation of the surrounding frontal, parietal and temporal opercular cortices.

The collation of the data obtained from the 6 islands is depicted in figure 2 . There appeared to be a pattern established by the somatomotor effects. It was possible to elicit movements of the face (figs. 6 G, H) by stimulation of all regions of the insula. These movements included muscular contractions of the lips, nose, eyelids and masticatory muscles. The facial movements tended to be homolateral and bilateral in relation to the excitation. In successive order, laryngeal, neck, upper extremity (figs, 6C, D), trunk, lower extremity (figs. 6E, F) and tail movements were obtained from the rostral to caudal expanse of the insula in both the dorsal and the ventral aspects of the island. The movements from the rostrodorsal bank of the insula tended to be bilateral and homolateral, while those from the caudoventral bank tended to be contralateral and bilateral. All of these movements were gross and similar to those characterized by Crosby ("56) as additional motor responses.

While a similarity between the dorsal and the ventral portions of the island was evident in the somatomotor responses obtained, there was a difference in the visceral behavior observed upon stimulation of the two regions. With electrical excitation at the limen, and extending to the caudal tip of the dorsorostral portion of the island, there were alterations in respiration, heart action, blood pressure and gastrointestinal tone, salivation and secretion of mucus (fig. 6G). During the stimulation and the placing of a destructive lesion, respiratory rate was slowed, labored, stertorous, and finally, apneic. Respiratory rate changes from 52 to 30 to 0 times per minute were accompanied by increased salivation and production of mucus. Laryngeal movements and secretory changes concomitant with respiration were visualized by means of a laryngoscope. 

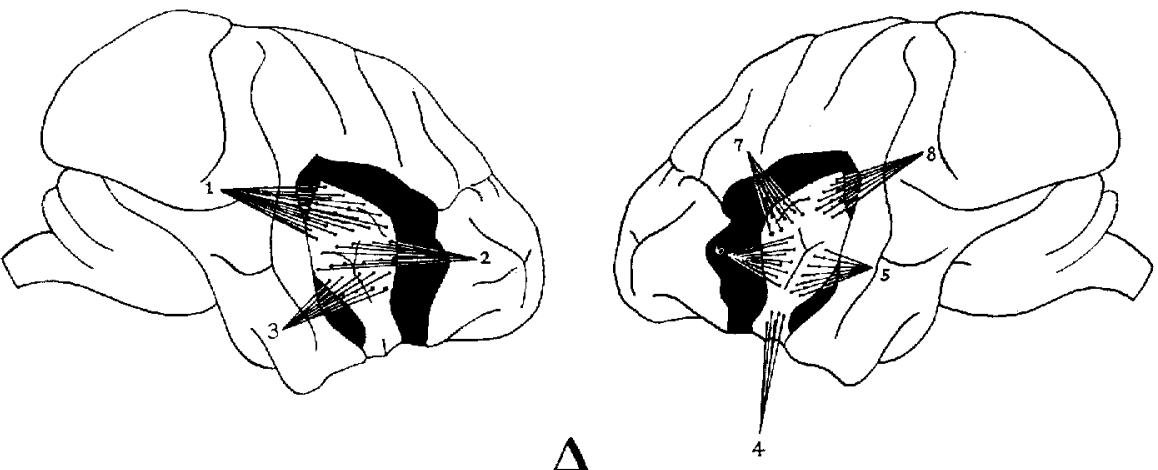

A
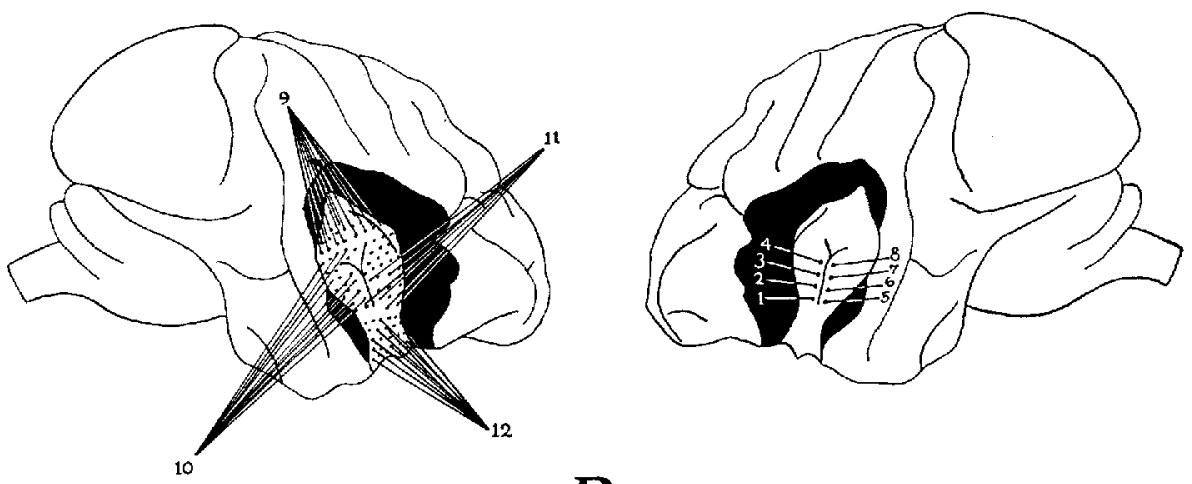

B
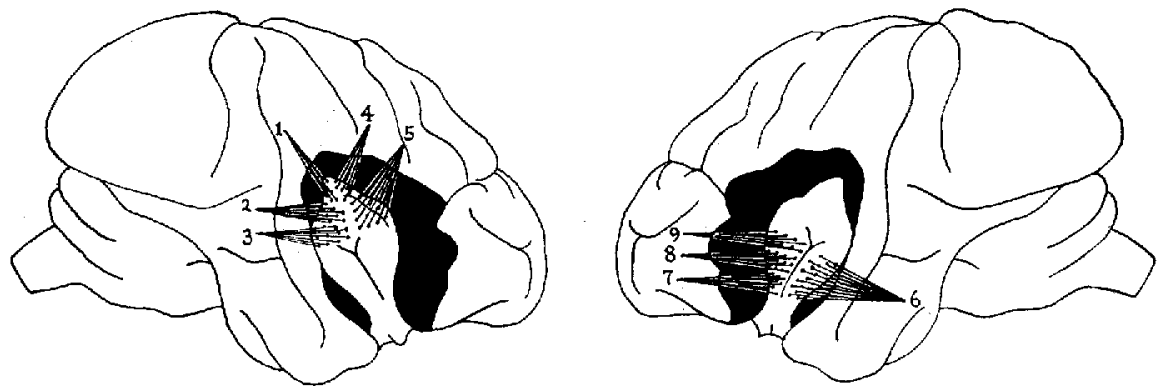

Q

Fig. 1 A, diagram of insular stimulation done on animal CLS2. B, diagram of insular stimulation done on animal CLS3. C, diagram of insular stimulation done on animal CLS4.

Cardiac rate was increased from a preoperative 100 pulsations per minute to a tachycardia exceeding 200 pulsations per minute during stimulation and destruc- tion of the dorsorostral insular cortex. Engorgement of cerebral blood vessels, increased edema of the brain and bleeding in the operative field suggested a rise in 


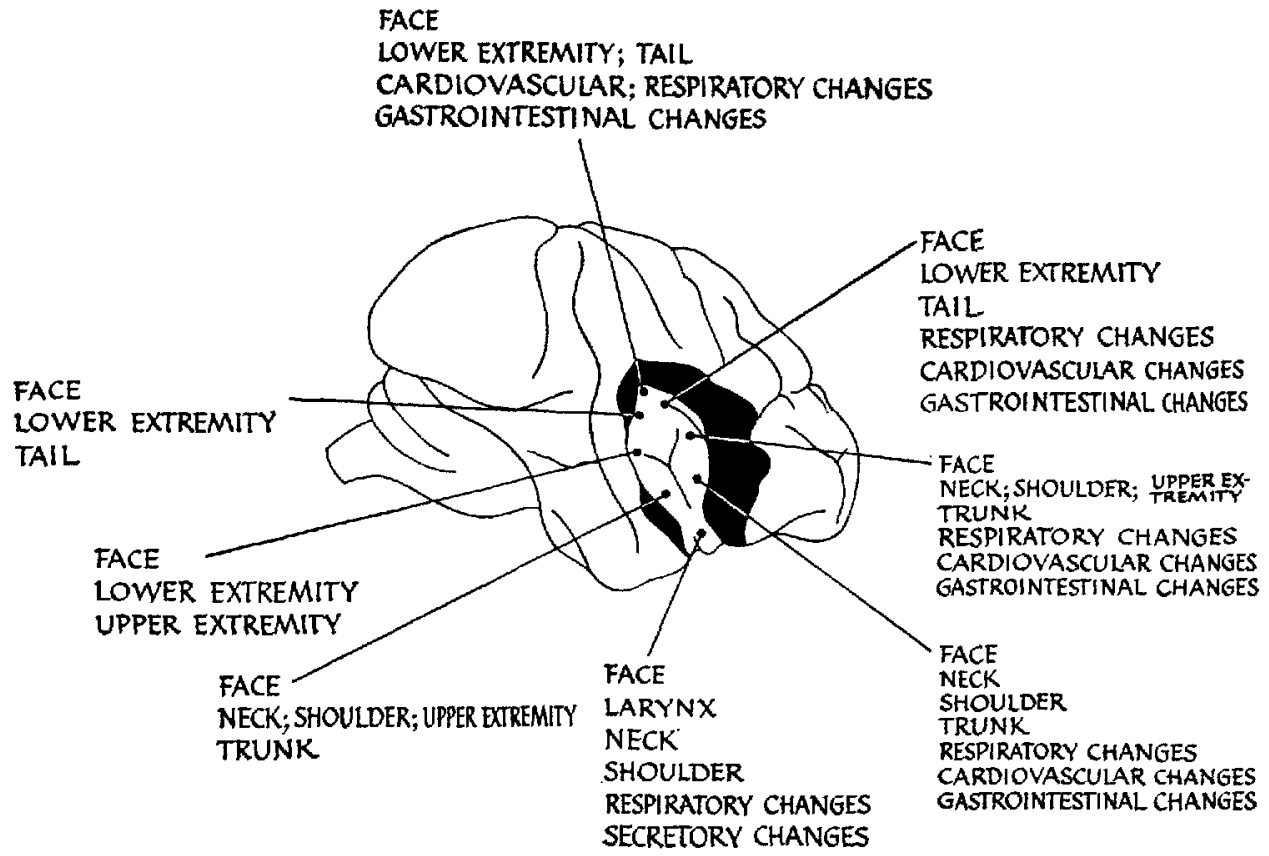

Fig. 2 Diagram summarizes somatovisceral responses from stimulation of the insula.

blood pressure at this time, also. Stimulation across the dorsorostral insula in the region giving rise to the somatic movement of the trunk musculature concomitantly produced an increase in the gastrointestinal motility which could be seen and felt through the abdominal wall. Simultaneous with the placing of a destructive lesion in this region, micturition and defecation to the extent of diarrhea occurred. Pupillary dilation (fig. 6G) appeared with excitation of the dorsorostral insular area toward the limen, and pupillary constriction with the stimulus applied at the more caudal part of this strip of cortex.

In the postoperative course of observation of two to 4 weeks, several features of altered behavior were present among the three animals. All three animals had a marked drop in body temperature which averaged $1.28^{\circ} \mathrm{C}$ following the experimental procedure. Piloerection occurred and was sustained for 4 weeks. Shivering was minimal, about 10 minutes, compared to that previously observed (Showers, '58, '59). Sweat tests performed on the animals showed a very slight increase in perspiration. The animals were sleepy for two to three days following the experiment. Sleep periods tended to alternate with hyperkinesia, and a lethargy was noticeable for 6 days in two instances.

All of the animals had marked oral movements without vocalization. Smacking of lips, protraction and retraction of the tongue, and sucking of the tongue against the palate were noticeable. These movements persisted throughout the observation period. Increased elimination was present in the animals, both in quantitative and periodic output following the experimentation. However, autopsy examination of the gastrointestinal mucosa did not reveal any gross changes. There was no increase in the food and water intake during the postoperative course.

\section{Microscopic study}

Examination of the microscopic slides prepared by Swank-Davenport ('35) modifcation of the Marchi technique gave equivocal evidence because the presence of injury to the regions of the three opercular cortices obscured the nature of degeneration from the lesions in the insula. Com- 
parison with previous work (Showers, '58, '59) seems to provide additive data for the course and the relations of certain intercortical pathways. Frontoparietal opercula of areas 6,4 and 1 and orbital gyrus 12 contributed fibers to the external and extreme capsules, corona radiata, superior longitudinal fasciculus and corpus callosum to distribute to adjacent frontal and parietal convolutions of the same and opposite sides (figs. 3A, 4, 5). Some fibers appeared to be directed into the insular cortex by the external capsule (fig. 5). A few fascicles extended into the cingulum toward the cingulate gyri (fig. 5). An equally substantial portion of degenerated fascicles entered capsules externa and extrema to extend downward into the inferior longitudinal fasciculus and to-
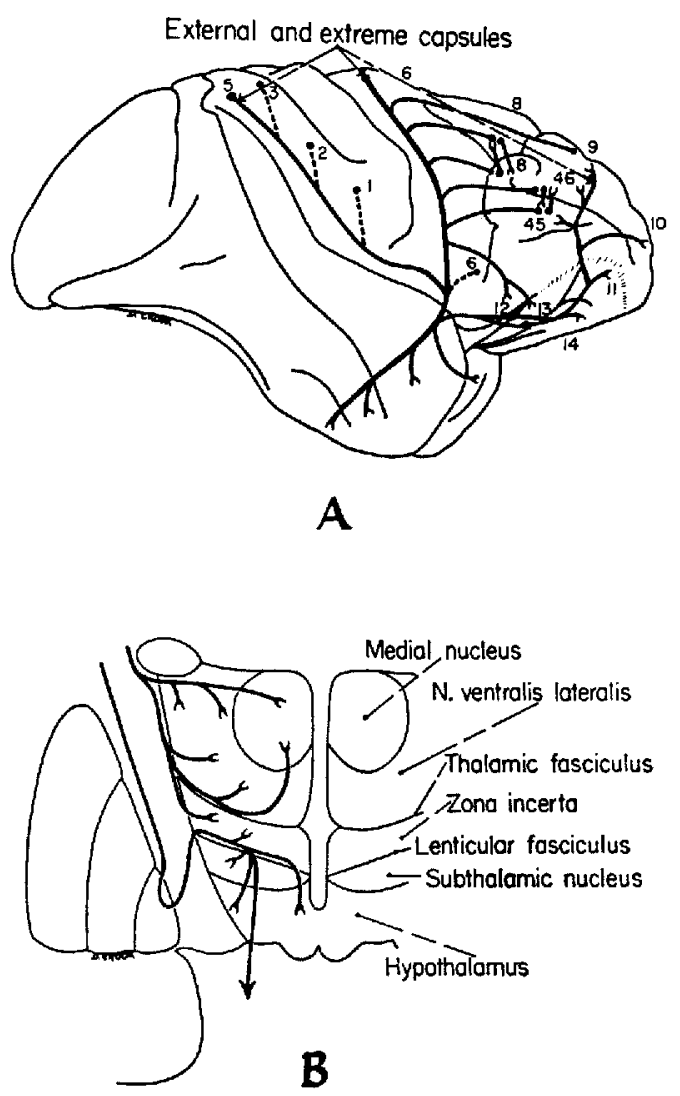

Fig. 3 A, diagram of frontal and parietal contributions to capsules externa and extrema. $B$, diagram of corticothalamic and corticotegmental pathways. ward inferior temporal and hippocampal gyri and the basolateral part of the amygdala. There is some transfer to the opposite hemisphere by way of the anterior commissure. Degenerating fascicles in the extreme capsule were followed into the superior and the middle temporal gyri. A diagram of these pathways is indicated in figures $3 \mathrm{~A}$ and 5 .

Those fibers from the degeneration of the lesion in the superior temporal gyrus, area 22 , were observed coursing downward through the inferior longitudinal fasciculus and anterior commissure to terminate in adjacent middle and inferior temporal gyri, hippocampus and amygdala. There was a suggestion that such fibers were directed upward into the capsules externa and extrema for distribution to the frontoparietal convolutions and insula. Variable degeneration appeared in the medial and the lateral septal nuclei, the diagonal band of Broca, the islands of Calleja and the substantia innominata. In their course to the olfactory tubercle and parolfactory area (precommissural

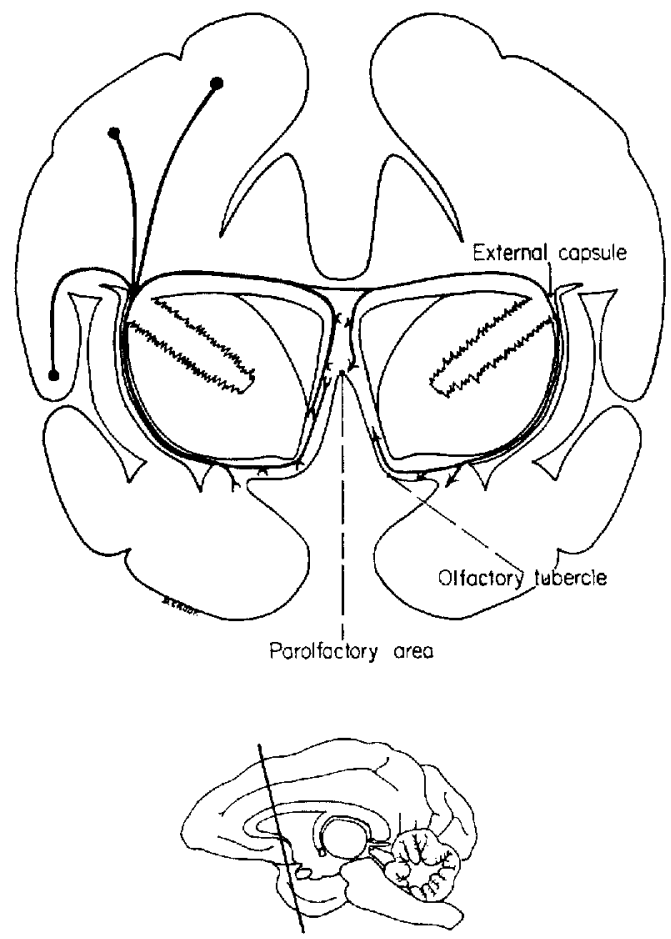

Fig. 4 Diagram of cortico-olfactory pathways. 
septum) the cortico-olfactory fibers, with or without crossing in the corpus callosum, passed in part through a dorsoventral association system (external capsule), then ventral and ventromedial to the lenticular nucleus to reach their destination. In part they accompanied the corpus callosum fibers medialward and, with or without crossing, turned ventralward near the midline to enter the parolfactory area.

In addition there was consistent degeneration into the medial geniculate nuclei on both sides in proportion to the size of the lesion in the superior temporal convolution. Mingled with corticogeniculate fibers were external corticotectal fascicles passing from the superior temporal convolution to the sublenticular part of the internal capsule and into the superior colliculus.

The terminal degeneration evident in the heads of the caudate nuclei, and traceable from them through the subcallosal bundles and the internal capsules into the corona radiata, seemed to have been derived from the opercular lesions. Similarly, there were opercular fibers which had entered the internal capsule to terminate in the red nucleus and surrounding tegmentum of the midbrain by way of the ventral peduncle of the lateral forebrain bundle (fig. 3B), or in the cerebral peduncle as corticobulbar, corticospinal and corticopontine components. A large number of fibers are transmitted through the internal capsule and the fibers of the comb bundle to end in the pars compacta and pars reticulata of the substantia nigra.

The lesions which intrude upon the island cortex are characterized by a smaller caliber of degeneration granules which stream directly across the capsule externa, claustrum and caspule extrema into putamen and globus pallidus. It is probable that some fascicles terminate in the claustrum. Most of the terminal degeneration is in the striatum and the nucleus accumbens, although some of the fascicles extend through the globus pallidus and below it as this region is traversed by the inferior thalamic peduncle (fig. 5). These latter fibers are dissipated as the nucleus ventralis lateralis, and nucleus medialis dorsalis of the dorsal thalamus appear in

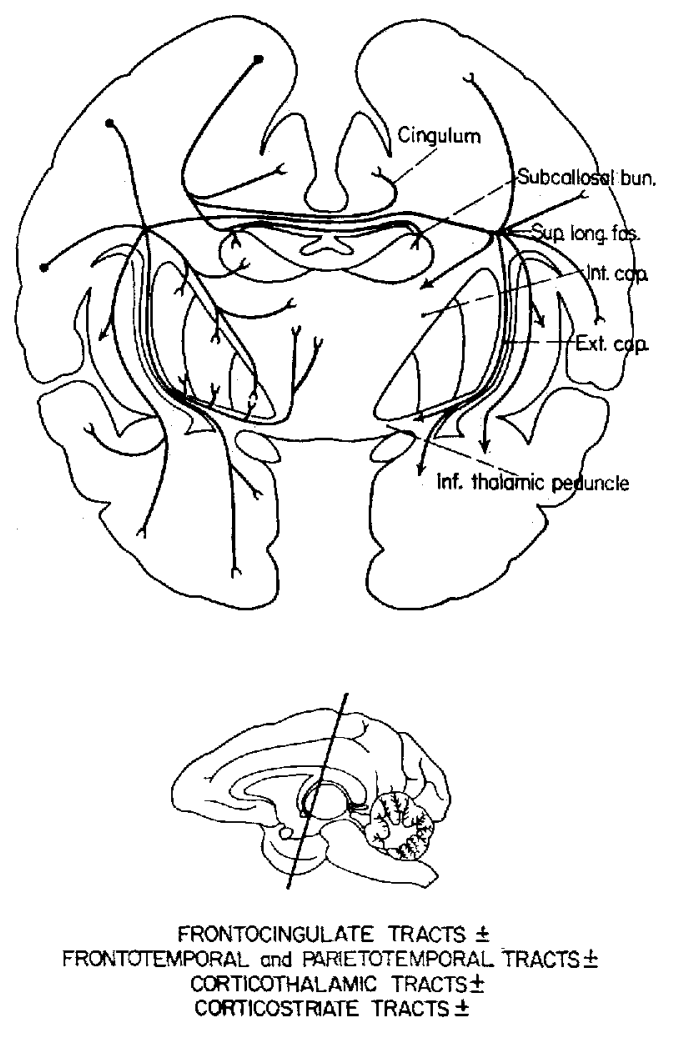

Fig. 5 Diagram of cortical association and commissural pathways.

the field (fig. 3B). Some fibers in the globus pallidus continue into the position of the pallidohypothalamic tract and disappear in the lateral hypothalamic area and the ventromedial hypothalamic nucleus.

In the animal CLS2 a terminal degeneration appeared in the habenular ganglion on the right side, at the ends of fibers which extend ventromedial to the lentiform nucleus and mingled with the fibers of the inferior thalamic peduncle to reach stria medullaris thalami and thence to the habenula. A few fibers extend through the habenular commissure to the contralateral ganglion. In this animal the lesion had injured the orbital gyrus of area 12 on the right side. Animal CLS3 has degeneration in the thalamic fasciculus and lenticular fasciculus, the former ending in nucleus ventralis lateralis, the latter in the nucleus of the field of Forel. 
The lesion had interrupted claustrum, putamen and the anterior commissure.

\section{DISCUSSION}

Neuroanatomical implications of somatovisceral results obtained by stimulation of the cingulate gyrus have been discussed in Showers ('59). There appears to be a considerable similarity between the experimental phenomena observed from both the insula and the cingulate gyrus. In each a dual representation of the body parts could be ascertained that was independent of the adjacent cerebral cortex. Increased strength of stimulation to each region blended all of the gross type of additional motor area movements into a generalized convulsive seizure. Visceral changes could be elicited from both of these cortical regions which involved a variety of adjustments to the environment with aspects of emotional reaction.

In this study, the microscopic data suggests that the insula is connected with the surrounding opercular cortex and orbital gyri by an extensive network of short association bands. The discharge pathway from the insula seems to extend directly across the external and the extreme capsules into the putamen and the globus pallidus. It would appear that these latter pathways are then contributing afferents to the lentiform nucleus, the efferent fibers of which, in turn, form the pallidohypothalamic tracts and the ansa and the fasciculus lenticularis. These connections would permit the insula to be linked with the visceral controls of the hypothalamus and the extrapyramidal system via the basal ganglia.

A conspicuous feature in the visceral responses of the dorsorostral portion of the insula was the presence of a sequence of oral, respiratory, and gastrointestinal movements along with increased salivary secretion. This has been referred to in dogs as a feeding pattern (Babkin and Van Buren, '51) and it was apparent, antecedent to epileptic seizures, in the clinical study done by Penfield and Faulk in "55.

\section{SUMMARY}

1. Analysis of 6 experiments on three macaque monkeys is described. Stimulation of the rostrodorsal and caudoventral portions of the insula gave a duplicate pattern of gross body movements. These included changes in facial expression, movements of upper extremities, trunk, lower extremities and tail in that order. This dual pattern was parallel in orientation and rostrocaudal in direction upon the insula.

2. Visceral changes in respiration, cardiovascular phenomena, oral secretions and gastrointestinal motility were included in the results of excitation of the rostrodorsal bank of the insula. Thus the insula could be termed an additional motor area and a cortical autonomic regulator with a convulsive seizure potential.

3 . Neuroanatomical connections described in this study included short association pathways from frontal, parietal and temporal opercula into the insula and an efferent pathway from the insula to the lentiform nucleus. The latter pathway interposes the island as a contributor to the extrapyramidal system and to the hypothalamic centers moderating visceral mechanisms.

4. Further documentation for vertically oriented association and commissural intercortical pathways of frontal, parietal and temporal cortices is presented.

\section{LITERATURE CITED}

Babkin, B. P., and J. M. Van Buren 1951 Mechanism and cortical representation of the feeding pattern. Arch. Neurol. Psychiat., 66: 1-19.

Bonin, G., and P. Bailey 1947 The Neocortex of Macaca mulatta. U. of Illinois Press, Urbana, 163 pp.

Crosby, E. C. 1956 The role of the midbrain as a part of the discharge path from higher centers. In: Progress in Neurobiology. J. Ariëns Kappers, ed. Elsevier Publishing Co., Amsterdam, pp. 217-231.

Frontera, J. G. 1955 Preliminary report on the results of electrical stimulation of island of Reil in the macaque. Anat. Rec., 121: 296.

1956 Some results obtained by electrical stimulation of the cortex of the island of Reil in the brain of monkey. J. Comp. Neur., 105: 365-394.

Grünbaum, A. S. F., and C. S. Sherrington 1903 Observations on the physiology of the cerebral cortex of the anthropoid apes. Proc. Roy. Soc. Lond., B., 72: 152-155.

Hoffman, B. L. 1951 An experimental study of the effects of electrical stimulation of the insular cortex on blood pressure, respiration and gastric motility in Macaca mulatta. Thesis, U. of Chicago, $65 \mathrm{pp}$. 
Hoffman, B. L., and T. Rasmussen 1953 Stimulation studies of insular cortex of Macaca mulatta. J. Neurophysiol., 16: 343-351.

Kaada, B, 1951 Somatomotor autonomic and electrocorticographic responses to electrical stimulation of rhinencephalic and other structures in primates, cat and dog. Acta Physiol. Scand., 24: (Sup. 83) 1-285.

Kaada, B., K. H. Pribram and J. Epstein 1949 Respiratory and vascular responses in mon keys from temporal pole, insula, orbital surface and cingulate gyrus. J. Neurophysiol., 12 : 347-356.

Leyton, A. S. F, and C. S. Sherrington 1917 Observations on the excitable cortex of the chimpanzee, orangutan, and gorilla. Quart. J. Exp. Physiol., 11: 135-222.

Livingstone, R. B. 1955 Some brain stem mechanisms relating to psychosomatic functions. Psychosomatic Med., 17: 347-354.

Penfield, W., and M. E. Faulk 1955 The insula, further observations on its function. Brain, 78: 446-470.

Pool, J. L. 1954 The visceral brain of man. J. Neurosurg., 11: 45-63.
Ruch, T. C., and H. D. Patton 1946 The relation of the deep opercular cortex to taste. Fed. Proc., 5: 89-90.

Showers, M. J. 1958 Correlation of medial thalamic nuclear activity with cortical and subcortical neuronal arcs. J. Comp. Neur., 109: 261-316.

- 1959 The cingulate gyrus: additional motor area and cortical autonomic regulator. Ibid., 112: 231-302.

Sugar, O., J. G. Chusid and J. D. French 1948 A second motor cortex in the monkey (Macaca mulatta). J. Neuropath. Exp. Neur., 7: 182189.

Swank, R. L., and H. A. Davenport 1935 Chlorate-osmic-formalin method for staining degenerated myelin. Stain Tech., 10: 87-90.

Walker, E. 1940 A cytoarchitectural study of the prefrontal area of the macaque monkey. J. Comp. Neur., 73: 59-86.

Wall, P. D., and G. Davis 1951 Three cerebral cortical systems affecting autonomic function. J. Neurophysiol., 14: 507-517.

\section{PLATE 1}

EXPLANATION OF FICURES

6A Right hemisphere of macaque dissected to show dorsorostral and ventrocaudal portions of insula. $\times 0.6$.

6B Left hemisphere of macaque showing cortical areas and outline of insula on opercular cortex. $\times 0.5$.

6C Animal CLS3 before stimulation of left insula.

6D Animal CLS3 after stimulation of left insula.

6E Animal CLS4 before stimulation of right insula.

6F Animal CLS4 after stimulation of right insula.

6G Animal CLS2 after stimulation of right insula. Note secretions from mouth and nose and left pupillary dilation.

6H Animal CLS3 during stimulation of left insula showing movement of face. $\mathrm{C}, \mathrm{D}, \mathrm{E}, \mathrm{F}, \mathrm{G}$, and $\mathrm{H}$ are enlargements of $16-\mathrm{mm}$ movie film. 

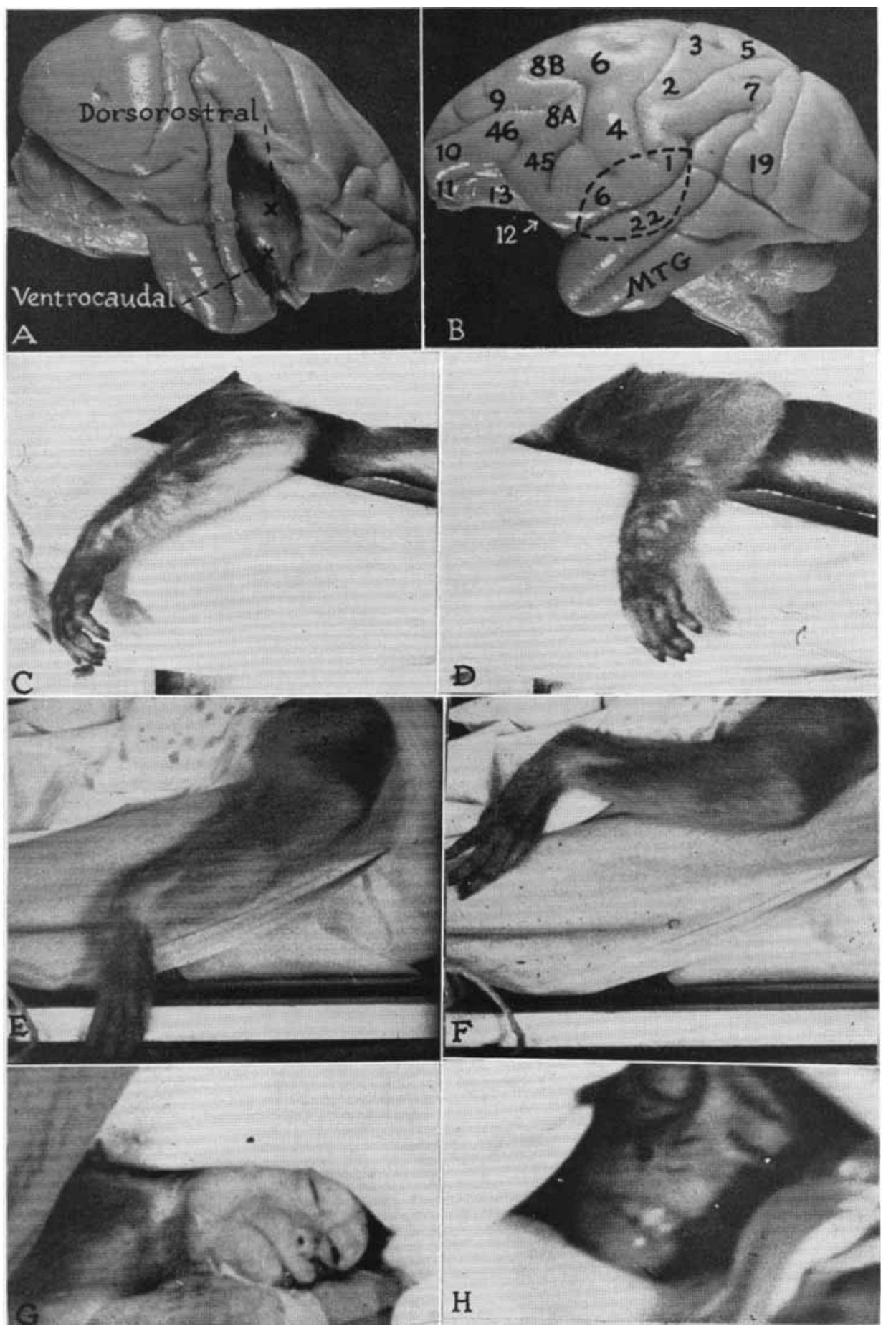